\title{
EXTRAÇÃO DE PIGMENTOS DAS SEMENTES DE Bixa orellana L.: UMA ALTERNATIVA PARA DISCIPLINAS EXPERIMENTAIS DE QUÍMICA ORGÂNICA
}

\author{
Charllyton Luis S. da Costa e Mariana H. Chaves* \\ Departamento de Química, Centro de Ciência da Natureza, Universidade Federal do Piauí, 64049-550 Teresina - PI
}

Recebido em 1/10/03; aceito em 19/5/04; publicado na web em 9/9/04

\begin{abstract}
EXTRACTION OF PIGMENTS FROM SEEDS OF Bixa orellana L.: AN ALTERNATIVE FOR EXPERIMENTAL COURSES IN ORGANIC CHEMISTRY. This paper describes methodologies for the extraction and characterization by TLC, UV-VIS, IR and NMR of bixin from Bixa orellana L. (urucum) seeds. Based on the results, the extraction with $\mathrm{NaOH} 5 \%$ is the fastest, uses lowcost materials, requires two to four laboratory hours and is a useful alternative for an experimental Organic Chemistry discipline.
\end{abstract}

Keywords: Bixa orellana; bixin; urucum.

\section{INTRODUÇÃO}

O uso de aditivos com a intenção de tornar os alimentos visualmente mais atraentes, seja na indústria alimentícia ou no uso doméstico cotidiano, é bastante comum. O corante extraído do pericarpo das sementes de urucum (Bixa orellana L.), um arbusto nativo do Brasil e de outras regiões tropicais do planeta, recebe a denominação internacional de annatto (E160b) ${ }^{1,2}$ sendo largamente utilizado em várias partes do mundo em escala industrial, por conferir coloração atraente a uma extensa gama de produtos manufaturados ${ }^{3}$.

O annatto (anato) é uma mistura de pigmentos de coloração amarelo-alaranjada em conseqüência da presença de vários carotenóides, com predominância absoluta de um atípico, conhecido como bixina. Os maiores produtores mundiais de anato são Peru, Brasil e Quênia ${ }^{1,2}$.

No Brasil, além do amplo emprego na indústria, a preparação comercial contendo $0,20-0,25 \%$ de bixina é conhecida como colorau, componente indissociável de inúmeros pratos da culinária brasileira. Este é produzido a partir das sementes de urucum, previamente aquecidas a $70{ }^{\circ} \mathrm{C}$ em óleo vegetal, seguido de abrasão com fubá ou farinha de mandioca ou pela mistura destas com urucum em pó, obtido por extração com solventes ${ }^{2,4}$.

A bixina (Figura 1) possui uma cadeia isoprênica de 24 carbonos, contendo um ácido carboxílico e um éster metílico nas extremidades, perfazendo assim a fórmula molecular $\mathrm{C}_{25} \mathrm{H}_{30} \mathrm{O}_{4}$. Representa $80 \%$ dos pigmentos da Bixa orellana L., ocorrendo apenas nesta espécie e em Aristolochia cymbifera Mart. ${ }^{2,5}$.

A bixina ocorre naturalmente na forma $16-Z$, porém durante o processo de extração é isomerizada conduzindo à forma $16-E$, denominada isobixina ${ }^{5}$.

Vários outros carotenóides $\left(\mathrm{C}_{19}, \mathrm{C}_{22}, \mathrm{C}_{24}, \mathrm{C}_{25}, \mathrm{C}_{30}, \mathrm{C}_{32}\right)$ foram isolados e identificados, porém constituem a parcela minoritária dos pigmentos. O constituinte majoritário das sementes de urucum é o geranilgeraniol, representando $1 \%$ das sementes secas ${ }^{6}$. A norbixina $\left(\mathrm{C}_{24} \mathrm{H}_{28} \mathrm{O}_{4}\right)$ é o derivado desmetilado da bixina que, apesar de ocorrer naturalmente, é quase sempre referida como produto da saponificação da bixina, sendo esta a sua forma de obtenção para fins comerciais ${ }^{1,7}$.

As preocupações relacionadas ao impacto da utilização de corantes sintéticos sobre a saúde direcionam as atenções para o uso daqueles de origem natural, pela crença de que estes sejam desprovidos de efeitos

*e-mail: mariana@ufpi.br

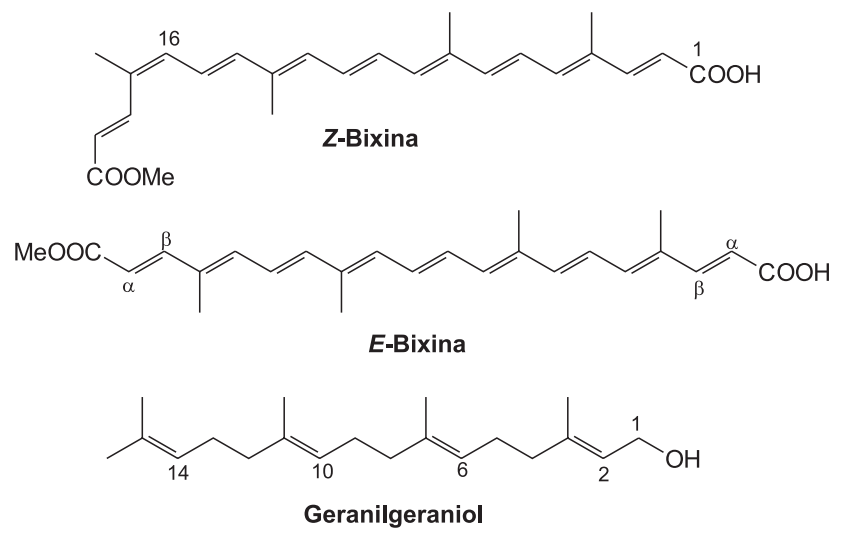

Figura 1. Substâncias presentes em sementes de Bixa orellana

tóxicos. A não exigência da apresentação de dados aprofundados referentes a análises toxicológicas e químicas para o registro de aditivos alimentares derivados de fontes naturais ${ }^{2,8}$, certamente torna as informações relativas a possíveis efeitos indesejáveis e/ou atividades farmacológicas decorrentes do seu uso, muito mais escassas do que se poderia prever diante da importância do tema. No Brasil, o uso do urucum é tão rotineiro que sua inocuidade sequer é questionada.

Estudos toxicológicos recentes demonstraram que a norbixina, e em última instância a bixina, uma vez que esta é desmetilada in vivo, é destituída de efeitos deletérios significativos sobre células hepáticas, renais e da medula óssea de camundongos e $\operatorname{ratos}^{2,9}$ e, embora nenhum efeito embriotóxico tenha sido demonstrado em $\operatorname{ratos}^{8}$, um grau considerável de interferência com o controle metabólico da glicose nestes roedores foi percebido ${ }^{2}$ e há relatos de uso popular da planta para tratamento do Diabetes mellitus ${ }^{8}$.

Os métodos de extração dos pigmentos da Bixa orellana L., seja para determinação da composição química ou averiguação de atividades sobre organismos vivos, são realizados com o uso de solventes, tais como propilenoglicol, óleo, água pura e soluções alcalinas ${ }^{10}$. Dependendo do solvente utilizado, a extração pode ser mais ou menos seletiva, de acordo com as conveniências de cada trabalho ${ }^{1,2,7}$. Todavia, o extrato obtido necessita passar por um processo de purificação utilizando-se técnicas, reagentes e equipamentos que tornam tal procedimento demorado, dispendioso e distante dos objetivos de uma disciplina de Química Orgânica até mesmo de cursos de graduação. 
Com a intenção de proporcionar uma alternativa prática, acessível e com o forte "apelo cotidiano" que possui o corante de urucum, uma vez que a proximidade do dia-a-dia tende a prender as atenções e despertar interesses, propomos a extração das sementes de Bixa orellana L. com uma solução de hidróxido de sódio, sob condições ordinárias e sem necessidade de instrumental específico.

Com exceção da norbixina, que ocorre naturalmente em pequenas quantidades, a bixina, pigmento majoritário do anato, é o único dos carotenóides isolados e identificados (Figura 1) que possui um grupo carboxílico livre ${ }^{1,5,7}$, sendo ele, portanto, um alvo potencial e seletivo para reação com $\mathrm{NaOH}$ e formação do sal da bixina, podendo a forma protonada ser reconstituída pela adição de um ácido.

A extração de sementes de urucum com $\mathrm{NaOH}$ é um procedimento rápido, de fácil execução e baixo custo, podendo ser executada em aulas práticas de Química Orgânica.

\section{PARTE EXPERIMENTAL}

\section{Procedimentos experimentais gerais}

Sementes de Bixa orellana L. (urucum) foram adquiridas no Mercado Central de Teresina em fevereiro de 2003. Os espectros de UVVis foram obtidos em um espectrofotômetro Hytachi U3000 na região de 900 a $190 \mathrm{~nm}$, utilizando solução de 20 ppm do extrato bruto em $\mathrm{CHCl}_{3}$. Os espectros de $\mathrm{RMN}{ }^{1} \mathrm{H}$ foram obtidos em espectrômetro Bruker Avance DRX-500 com o uso de $\mathrm{CDCl}_{3}$ como solvente e TMS como padrão de referência interno. $\mathrm{O}$ espectro na região do Infravermelho foi obtido em espectrômetro Bolmem FT-IR, modelo MB-102 utilizando partilhas de $\mathrm{KBr}$. As placas cromatográficas foram preparadas com camadas de $0,25 \mathrm{~mm}$ de gel de sílica $60 \mathrm{da}$ Vetec, eluídas em hexano-acetato de etila (8:2) e $\mathrm{CHCl}_{3}-\mathrm{MeOH}$ (9:1). Os cromatogramas foram observados no visível antes e após as placas serem borrifadas com solução de sulfato cérico seguido de aquecimento.

\section{Extração das sementes de urucum}

Método 1: duas amostras de sementes de urucum (10 g) foram submetidas a extração, uma com acetona $(40 \mathrm{~mL})$ e outra com hexano $(40 \mathrm{~mL})$, à temperatura ambiente, por um período de $30 \mathrm{~min}$, sob agitação freqüente. Separou-se as sementes por filtração e evaporouse os solventes em evaporador rotativo.

Método 2: sementes de urucum (10 g) foram submetidas a extração com acetona em aparelhagem Soxhlet por um período de 1,5 h. Removeram-se as sementes por filtração e evaporou-se o solvente em evaporador rotativo.

Método 3: duas amostras de sementes de urucum (10 g) foram submetidas a extração, uma com $40 \mathrm{~mL}$ de solução de $\mathrm{NaOH} 5 \%$ por 5 min e a outra com $40 \mathrm{~mL}$ de $\mathrm{NaHCO}_{3} 10 \%$ por $30 \mathrm{~min}$, sob agitação freqüente. Separaram-se as sementes por filtração, adicionou-se $\mathrm{HCl}$ concentrado à mistura alcalina até $\mathrm{pH} 4$, filtrou-se usando papel de filtro previamente pesado, lavou-se o sólido com água destilada até o $\mathrm{pH}$ neutro e secou-se o extrato em estufa a $70{ }^{\circ} \mathrm{C}$ por $30 \mathrm{~min}$.

\section{Caracterização da bixina}

Os extratos de semente de urucum foram inicialmente analisados por cromatografia em camada delgada comparativa (CCDC) usando como eluentes hexano-acetato de etila (8:2) e clorofórmio-metanol (9:1). Cerca de $5 \mathrm{mg}$ de cada extrato foram dissolvidos em $1 \mathrm{~mL}$ de $\mathrm{H}_{2} \mathrm{SO}_{4}$ concentrado para verificação da presença de carotenóides ${ }^{10}$. Aproximadamente $30 \mathrm{mg}$ dos extratos obtidos com solução de $\mathrm{NaOH}$ e com acetona foram analisados por $\mathrm{RMN}{ }^{1} \mathrm{H} \mathrm{e}{ }^{13} \mathrm{C}$, usando $\mathrm{CDCl}_{3}$ como solvente.

Os extratos das sementes de urucum $(10 \mathrm{mg})$ obtidos por extração com acetona à temperatura ambiente, acetona em Soxhlet e com $\mathrm{NaOH}$ foram dissolvidos em clorofórmio e as soluções resultantes tiveram seus volumes acertados para $100 \mathrm{~mL}$ com o mesmo solvente. Uma alíquota de $10 \mathrm{~mL}$ de cada solução foi retirada e diluída até $50 \mathrm{~mL}$ com $\mathrm{CHCl}_{3}$, obtendo-se três soluções com concentração de $20 \mathrm{mg} / \mathrm{L}$. Estas soluções foram analisadas no espectrofotômetro UV-Vis entre 900 e $190 \mathrm{~nm}$ e os respectivos espectros foram registrados e comparados com os da literatura ${ }^{3}$. A partir dos espectros obtidos determinou-se a concentração de bixina utilizando a expressão $A=a c b$, onde $\boldsymbol{A}$ representa a absorbância da solução clorofórmica do extrato, lida no espectrofotômetro, $c$ é a concentração de bixina na solução $(\mathrm{g} / \mathrm{L}), \boldsymbol{b}$ é o caminho ótico $(1 \mathrm{~cm})$ e $\boldsymbol{a}$ é o coeficiente de absorção da bixina em $\mathrm{CHCl}_{3}$ (2826) no $\lambda_{\text {max }} 470 \mathrm{~nm}$ descrito por Mercadante $^{4}$. O rendimento de bixina foi determinado considerando-se a massa do extrato obtida em cada extração.

A relação bixina/geranilgeraniol foi determinada dividindo-se o valor da integração de um dos sinais de $\mathrm{H}-\beta$ aos grupos carboxílicos da bixina pelo valor da integração do sinal de H-1 do geranilgeraniol e multiplicando-se por $2^{12}$.

\section{RESULTADOS E DISCUSSÃO}

A extração das sementes de $B$. orellana teve como finalidade escolher qual seria o melhor solvente para a extração da bixina. $\mathrm{O}$ hexano e a solução de $\mathrm{NaHCO}_{3}$ a $10 \%$ não se mostraram eficientes, os rendimentos obtidos foram 0,22 e $1,56 \%$, respectivamente (Tabela 1 e Figura 2). Os solventes que se mostraram mais eficientes foram acetona em Soxhlet e a solução de $\mathrm{NaOH} 5 \%$. Apesar do rendimento de extrato bruto obtido com acetona em Soxhlet ter sido o maior $(6,16 \pm 0,28)$, o rendimento de bixina, determinado por espectrometria UV-Vis neste extrato $(1,31 \pm 0,43 \%)$, foi menor que o determinado no extrato bruto preparado com solução de $\mathrm{NaOH} 5 \%$ $(3,10 \pm 0,09 \%)$, conforme apresentado nas Figuras 2 e 3 e Tabela 1. Além disso, outras substâncias extraídas com acetona podem afetar a quantificação da bixina, e ainda, o tempo gasto na extração com acetona em Soxhlet foi superior ao da extração com solução de $\mathrm{NaOH}$ $5 \%$. Vale ressaltar que outros carotenóides podem ser detectados

Tabela 1. Resultados obtidos na extração das sementes de Bixa orellana por diferentes solventes

\begin{tabular}{|c|c|c|c|c|c|c|}
\hline Método de extração & $\begin{array}{c}\text { Massa de } \\
\text { sementes/g }\end{array}$ & $\begin{array}{c}\text { Tempo de } \\
\text { extração/min }\end{array}$ & $\begin{array}{c}\text { Massa } \\
\text { extraída/mg }\end{array}$ & $\begin{array}{c}\text { Rendimento } \\
\text { da extração/\% }\end{array}$ & $\begin{array}{l}\text { Rendimento } \\
\text { de bixina/\%* }\end{array}$ & $\begin{array}{l}\text { Relação bixina/ } \\
\text { geranilgeraniol }\end{array}$ \\
\hline Acetona & 10 & 30 & $498,67 \pm 5,86$ & $4,99 \pm 0,06$ & $1,18 \pm 0,45$ & $1: 3$ \\
\hline Hexano & 10 & 30 & 22 & 0,22 & ND & ND \\
\hline Acetona (Soxhlet) & 10 & 90 & $616 \pm 28,28$ & $6,16 \pm 0,28$ & $1,31 \pm 0,43$ & $1: 3$ \\
\hline $\mathrm{NaOH} 5 \%$ & 10 & 5 & $493,07 \pm 31,87$ & $4,93 \pm 0,32$ & $3,10 \pm 0,09$ & $1: 2$ \\
\hline $\mathrm{NaHCO}_{3} 10 \%$ & 10 & 30 & 156 & 1,56 & ND & ND \\
\hline
\end{tabular}

*Determinado por UV-Vis em relação à massa de extrato; ND: não determinado; $\$$ determinada por $\mathrm{RMN}{ }^{1} \mathrm{H}$. 


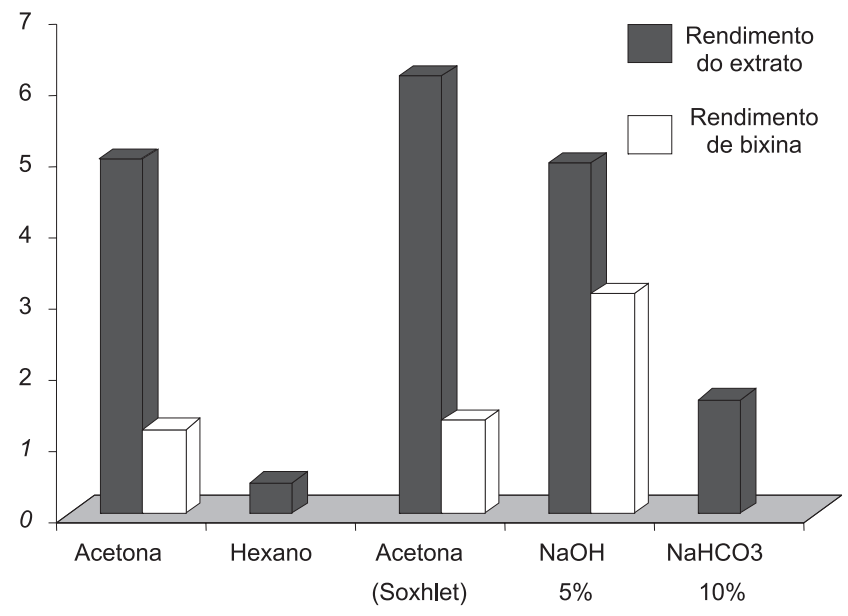

Figura 2. Gráfico da porcentagem de extratos e de bixina das sementes de Bixa orellana obtidos com diferentes solventes

concomitantemente com a bixina na análise por UV-Vis, contudo, como mencionado anteriormente, a ocorrência desta nas sementes de urucum é da ordem de $80 \%$ do total dos pigmentos existentes ${ }^{2}$.

A literatura registra uma concentração média de bixina nas sementes de urucum variando entre $1,2-2,3 \%$, no entanto, o rendimento pode estar sujeito ao tipo de solvente usado na extração e a fatores ambientais, tais como temperatura, iluminação, índice pluviométrico e tipo de solo $^{5,10}$. Os resultados obtidos permitiram eleger a solução de $\mathrm{NaOH} 5 \%$ como o solvente ideal para a obtenção do extrato.

$\mathrm{O}$ tratamento do extrato com $\mathrm{H}_{2} \mathrm{SO}_{4}$ concentrado resultou no desenvolvimento de coloração azul esverdeada, característica da presença de carotenóides ${ }^{11}$.

A realização de CCDC usando como eluente hexano-acetato de etila (8:2) teve por finalidade a verificação da existência de constituintes menos polares nos extratos, enquanto que o uso de $\mathrm{CHCl}_{3}$ $\mathrm{MeOH}$ (9:1) foi para eluição dos constituintes mais polares, como a bixina. Para o primeiro cromatograma obtido observou-se que apenas uma pequena parcela do material foi eluído, resultando em uma mancha uniforme, de formato alongado, coloração laranja, com $R_{f}$ abaixo de 0,5 e que sugere ser de carotenóides com grupamento ácido. Este resultado permite ainda deduzir que carotenóides menos polares, tipo $\beta$-caroteno, devem estar presentes em pequenas quantidades, uma vez que não se observou manchas com características deste tipo de substâncias. O segundo cromatograma mostrou que grande parte do material foi eluído, originando manchas de formato alongado, coloração laranja, distribuídas por toda a placa, ficando a zona de maior concentração com $\mathrm{R}_{\mathrm{f}}$ de aproximadamente 0,4 e coloração vermelho-alaranjada, que sugere ser a bixina.

Os espectros de UV-Vis (Figura 3) dos extratos de urucum mostraram-se semelhantes aos relatados para a bixina ${ }^{3}$.

A presença da bixina nos extratos acetônicos e em solução de $\mathrm{NaOH} 5 \%$ foi comprovada através da análise dos espectros de RMN ${ }^{1} \mathrm{H}$ mediante observação de dois pares de dubletos em $\delta 5,89 /$ $7,98(\mathrm{~J}=15,8 \mathrm{~Hz})$ e $\delta 5,93 / 7,47(\mathrm{~J}=15,5 \mathrm{~Hz})$ atribuídos, respectivamente, a $\mathrm{H}-\alpha / \mathrm{H}-\beta$ aos grupos carboxílicos do ácido livre e do éster, juntamente com o singleto em $\delta 3,80$ de metoxila de éster ${ }^{5}$. O espectro de $\mathrm{RMN}{ }^{13} \mathrm{C}$ também confirmou a presença da bixina, pelos sinais de carbono da metoxila de éster $(\delta 51,6)$, e dois sinais em $\delta 171,8$ e 168,0 referentes às carbonilas de ácido e éster, respectivamente ${ }^{5}$.

Os espectros de RMN dos extratos acetônicos e em solução de $\mathrm{NaOH} 5 \%$ mostraram que o diterpenóide geranilgeraniol foi o componente majoritário das sementes de urucum, conforme relatado na

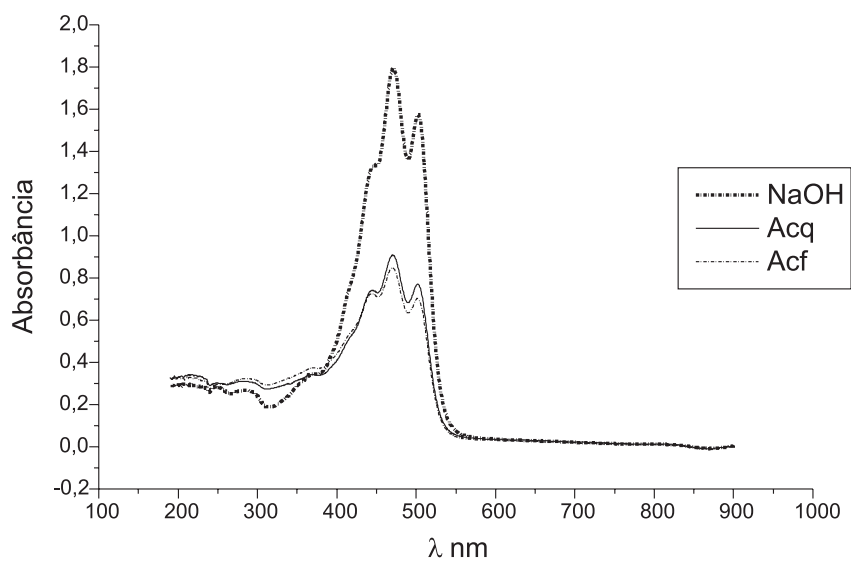

Figura 3. Espectro UV-Vis das soluções de extrato das sementes de Bixa

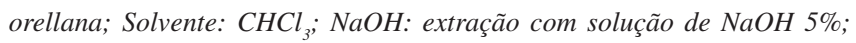
Acq.: extração com acetona em Soxhlet; Acf.: extração com acetona a frio

literatura ${ }^{6}$. Os sinais observados no espectro de $\mathrm{RMN}{ }^{1} \mathrm{H}$ que caracterizam o geranilgeraniol (Figura 4) são: $\delta 4,18(\mathrm{~d}, 6,7 \mathrm{~Hz}, \mathrm{H}-1), \delta$ 5,43 (tl, 6,8 Hz, H-2), $\delta$ 5,12 (sl, H-6, H-10 e H-14), $\delta$ 1,62 (s, 3CH ) $^{2}$ e $\delta 1,70\left(\mathrm{~s}, 2 \mathrm{CH}_{3}\right)$, contudo, no espectro de $\mathrm{RMN}{ }^{13} \mathrm{C}$ os sinais característicos para esta substância ocorrem em $\delta 59,4\left(\mathrm{CH}_{2}, \mathrm{C}-1\right), \delta 139,9$, $135,4,134,0$, e 131,3 (carbonos olefínicos não hidrogenados), $\delta 124,4,124,2,123,7$ e 123,2 (carbonos metínicos olefínicos) e os situados entre $\delta 39,7-16,0$ (carbonos saturados não oxigenados) ${ }^{6,13}$.

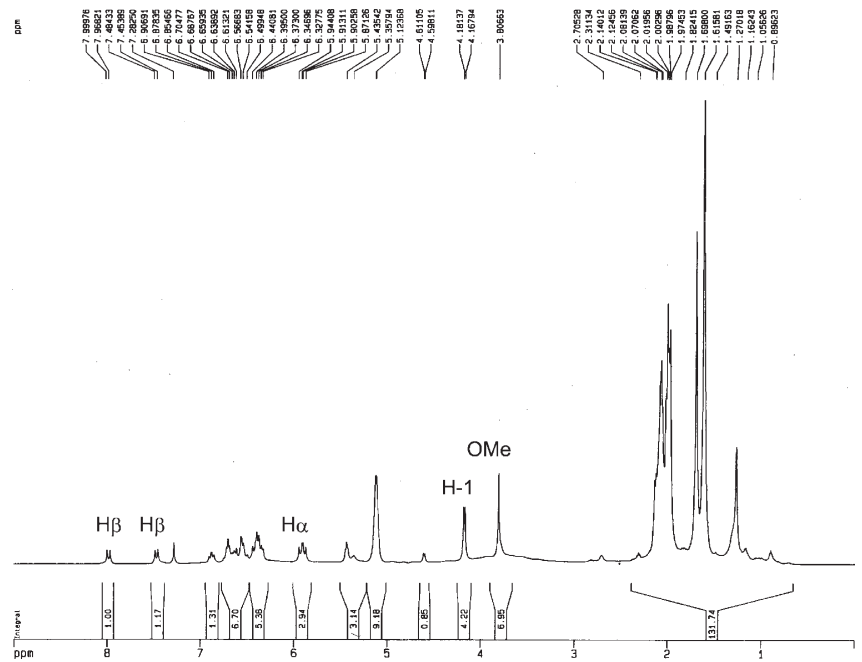

Figura 4. Espectro de $R M N{ }^{1} \mathrm{H}$ do extrato de sementes de Bixa orellana em solução de $\mathrm{NaOH} 5 \%\left(\mathrm{CDCl}_{3}, 500 \mathrm{MHz}\right)$

A relação bixina/geranilgeraniol, determinada através do espectro de $\mathrm{RMN}{ }^{1} \mathrm{H}$, foi 1:3 nos extratos acetônicos e 1:2 no extrato preparado com solução de $\mathrm{NaOH}$ (Tabela 1). Estes resultados foram compatíveis com o percentual de bixina determinada por UV-Vis nos extratos, considerando-se que devem existir outras substâncias além da bixina e geranilgeraniol no extrato de urucum.

$\mathrm{O}$ espectro na região do infravermelho (IV) do extrato obtido com solução de $\mathrm{NaOH} 5 \%$ de sementes de urucum (Figura 5) apresenta banda larga e forte na região de $3500-2500 \mathrm{~cm}^{-1}$ de estiramento $\mathrm{O}-\mathrm{H}$ de álcool, atribuído ao geranilgeraniol, sobreposta à banda de 
estiramento O-H de ácido. Esta última juntamente com a banda forte de estiramento de carbonila de éster $\alpha, \beta$-insaturado $\left(1717 \mathrm{~cm}^{-1}\right)$ contendo um ombro que sugere ser de outra carbonila, são indicativos da presença de bixina. $\mathrm{O}$ espectro apresenta ainda, entre outras, bandas de estiramento $\mathrm{C}=\mathrm{C}\left(1608,1600\right.$ e $\left.1563 \mathrm{~cm}^{-1}\right), \mathrm{C}-\mathrm{H} \mathrm{sp}{ }^{3}(2856$, 2919 e $\left.2969 \mathrm{~cm}^{-1}\right), \mathrm{C}-\mathrm{H} \mathrm{sp}{ }^{2}\left(3031 \mathrm{~cm}^{-1}\right), \mathrm{C}-\mathrm{O}$ de álcool primário $\left(1161 \mathrm{~cm}^{-1}\right)$ e deformação C-H de olefinas $\left(1006\right.$ e $\left.964 \mathrm{~cm}^{-1}\right)$. Estes dados mostraram-se compatíveis com os relatados na literatura para as duas substãncias ${ }^{5,6}$.

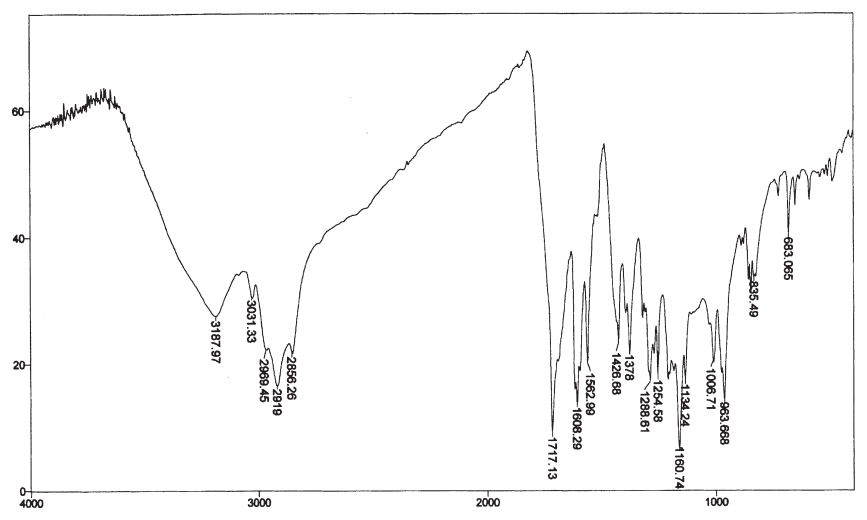

Figura 5. Espectro na região do infravermelho do extrato de sementes de Bixa orellana

A extração do geranilgeraniol, uma substância neutra, da semente de urucum, com solvente quimicamente ativo pode ser questionada, porém, este fato pode ser justificado considerando que esta substância foi arrastada, por ação de lavagem, pela solução de hidróxido de sódio $^{10}$.

O experimento da forma como está sendo proposto neste trabalho, iniciando-se com a extração seguida de análise por UV-Vis, RMN e IV, poderá ser executado em aulas de Química Orgânica e Química Analítica de cursos de graduação. Opcionalmente, quando não se dispuser do equipamento, poderá ser omitida a análise por RMN, pois a espectrometria na região do infravermelho permite confirmar a presença de bixina e geranilgeraniol no extrato.

A simples extração das sementes com determinação do rendimento, como forma de adaptação às condições locais, poderá ser realizada até mesmo em aulas do ensino médio, usando materiais alternativos como soda cáustica e álcool ou acetona comerciais.

Além dos cálculos sobre o rendimento da bixina e a relação bixina/geranil-geraniol, alguns questionamentos poderão ser levantados para discussão em sala, tais como o porquê da bixina ter sido extraída em maior concentração com solução de $\mathrm{NaOH}$; as razões pelas quais a bixina absorve luz com $\lambda_{\text {max }} 470 \mathrm{~nm}$; a cor observada para bixina, considerando a cor e o comprimento de onda da luz absorvida $^{14}$ e as razões de se observar coloração azul pelo tratamento do extrato de urucum com ácido sulfúrico concentrado ${ }^{15}$.

\section{CONCLUSÃO}

O método de extração de sementes de urucum com solução de hidróxido de sódio descrito neste trabalho conduziu ao melhor rendimento de bixina quando comparado à extração com acetona, à temperatura ambiente e em Soxhlet, e permite sua implementação em disciplinas que dispõem de períodos curtos de aula, ficando a caracterização da bixina por UV-Vis, RMN e IV e a discussão para serem realizadas em aulas posteriores. Além disso, somente a extração não necessita de instrumentação sofisticada, podendo ser realizada inclusive no ensino médio utilizando materiais de baixo custo e de fácil aquisição. De forma opcional, o experimento pode ser montado incluindo também a extração com acetona à temperatura ambiente e em Soxhlet, a ser executado por diferentes equipes, com o objetivo de comparar os rendimentos e com enfoque na discussão dos conceitos de extração contínua e maceração com solvente quimicamente ativo.

\section{AGRADECIMENTOS}

À CAPES/PROCAD pelo apoio financeiro e bolsa de C. L. S. da Costa; ao Centro Nordestino de Aplicação e Uso da RMN (CENAUREMN/UFC) pela execução dos espectros.

\section{REFERÊNCIAS}

1. Mercadante, A. Z.; Steck, A.; Rodrigues-Amaya, D.; Pfander, H.; Briton, G; Phytochemistry 1996, 41, 1201.

2. Fernandes, A. C. S.; Almeida, C. A.; Albano, F.; Laranja, G. A. T.; Felzenszwalb, I.; Lage, C. L. S.; de Sá, C. C. N. F.; Moura, A. S.; Kovary, K.; J. Nutr. Biochem. 2002,13, 411; Araújo, J. A.; Química de alimentos: teoria e prática, 2a. ed., Ed. UFV: Viçosa, 1999.

3. Oliveira, L. F. C.; Dantas, S. O.; Velozo, E. S.; Santos, P. S.; Ribeiro; M. C. C.; J. Mol. Struct. 1997, 435, 101.

4. Mercadante, A. Z.; Tocchini, L.; Ciênc. Tecnol. Aliment. 2001, 21, 310.

5. Sousa, P. S.; Matos, M. E. O.; Matos, F. J. A.; Machado, M. I. L.; Craveiro, A. A.; Constituintes químicos ativos de plantas medicinais brasileiras, Ed. UFC: Fortaleza, 1991.

6. Jondiko, I. J. O.; Pattenden, G.; Phytochemistry 1989, 28, 3159.

7. Mercadante, A. Z.; Steck, A.; Pfander, H.; Briton, G.; Phytochemistry 1997, 46, 1379; Mercadante, A. Z.; Steck, A.; Pfander, H.; Briton, G; Phytochemistry 1999, 52, 135.

8. Paumgartten, F. J. R.; De-Carvalho, R. R.; Araújo, I. B.; Pinto, F. M.; Borges, O. O.; Souza, C. A. M.; Kuriyama, S. N.; Food Chem. Toxicol. 2002, 40, 1595 .

9. Lima, R. O. A.; Azevedo, L.; Ribeiro, L. R.; Salvadori, D. M. F.; Food Chem. Toxicol. 2003, 41, 189.

10. Shuhama, I. K.; Aguiar, M. L.; Oliveira, W. P.; Freitas, L. A. P.; J. Food Engin. 2003, 59, 93

11. Ikan, R.; Natural Products: A Laboratory Guide, $2^{\text {nd }}$ ed., Academic Press: San Diego, 1991.

12. Chaves, M. H.; Barbosa, A. S.; Moita Neto, J. M.; Aued-Pimentel, S.; Lago, J. H. G.; Quim. Nova 2004, 27, 404.

13. Rahman, A.; Ahmad, V. U.; ${ }^{13}$ C of Natural Products: Diterpenes, Plenum Press: New York, 1992.

14. Pavia, D. L.; Lampman, G. M.; Kriz, G. S.; Introduction to Spectroscopy, $3^{\text {rd }}$ ed., Brooks/Cole: Australia, 2001.

15. MacBeath, M. E.; Richardson, A. L.; J. Chem. Educ. 1986, 63, 1093. 